\title{
Functional magnetic resonance imaging for brain mapping in neurosurgery
}

\author{
Paul E. Kim, M.D., And Manbir Singh, Ph.D. \\ Departments of Neuroradiology and Biomedical Engineering, Keck School of Medicine, University of \\ Southern California, Los Angeles, California
}

\begin{abstract}
One of the most pertinent applications of the principle primum non nocere (first do no harm) is in the optimization of neurosurgical procedures for patients with resectable lesions. The gold standard for identifying eloquent areas of the brain to be avoided in resections is direct cortical stimulation and somatosensory evoked potential monitoring, which is itself an invasive, cumbersome and difficult technique for mapping these areas. Functional magnetic resonance imaging shows great promise as a viable noninvasive alternative to invasive mapping as well as significant current clinical utility in cases in which it cannot yet fully supplant cortical stimulation methods. Ongoing work is directed toward overcoming technical limitations, improved mapping of complex functions such as language and memory, and mapping of white matter tracts.
\end{abstract}

\section{KEY WORDS • blood oxygen level-dependent functional magnetic resonance imaging • brain mapping • neuronavigation}

The goal of modern neurosurgery is to improve the survival rates and quality of life of patients with surgically treatable intracranial lesions. It is known, however, that tumor progression and survival in cases of malignant intracranial tumors is contingent on the extent of tumor removal. The protection of functions potentially at risk during surgery is facilitated by functional mapping of critical eloquent areas. The gold standards for mapping eloquent areas of the brain are invasive cortical stimulation and somatosensory evoked potential monitoring.

A number of secondary physiological changes occur as a result of neuronal activity in the brain. Measurable changes such as glucose utilization led to the development of functional mapping with ${ }^{18}$ FDG-PET. It was also found that another of these changes, local differences in oxygen use, could be demonstrated and localized using MR imaging, which led to the development of BOLD fMR imaging. The introduction of frameless intraoperative neuronavigation systems such as StealthStation (Medtronic, Boulder, CO) or Vector Vision (BrainLab AG, Heimstetten, Germany) has allowed the precise coregistration and transfer of fMR imaging data into the surgical field. Understandably, however, the acceptance of MR imaging by neurobiologists in the investigation of neurophysiological functions of the human cerebral cortex was quick relative to its acceptance as a clinical tool by neurosurgeons; the

Abbreviations used in this paper: BOLD = blood oxygen leveldependent; FDG $=\left[{ }^{18}\right.$ F fluoro-2-deoxy-D-glucose; fMR = functional magnetic resonance; $\mathrm{PET}=$ positron emission tomography; $3 \mathrm{D}=$ three-dimensional. stakes are obviously different. Although the status of fMR imaging currently is investigational; it is an evolving technology that shows both future promise and present-day utility. In some instances, it has been used successfully in lieu of invasive mapping tools. Indeed, in certain cases, evidence exists that fMR imaging mapping may be more appropriate than cortical stimulation. ${ }^{13}$ Its shortcomings can be categorized into the following two broad categories: 1) technical, which will be at the very least partially overcome by ongoing technical advancement; and 2) neuroscientific understanding in general, which otherwise applies to all modalities of brain mapping (including direct cortical stimulation and somatosensory evoked potential monitoring). When not used as an outright substitute for invasive mapping, fMR imaging has a well-documented adjunctive role in optimizing surgical procedures and outcomes that belie its investigational status. ${ }^{17,26,43}$

\section{TECHNICAL BACKGROUND OF FMR IMAGING}

The feasibility of using MR imaging for mapping of neuronal activation with the BOLD technique was first demonstrated in the human brain in 1992 by Kwong, et al., ${ }^{23}$ and Ogawa, et al. ${ }^{30}$ who used a simple visual perception task, and by Bandettini, et al., ${ }^{2}$ who used a motor task. Since then, several investigators have described the use of fMR imaging for presurgical mapping, many of whom correlated their finding with invasive cortical mapping data. ${ }^{1,12,14,24,25,28,32,35,37,47}$

Like other functional neuroimaging modalities such as PET, BOLD fMR imaging is based on secondary physio- 
logical responses to brain activation. Neuronal activity initially causes a transient increase in oxygen extraction, which is quickly followed by compensatory vasodilation and resultant net overall increase in blood oxygen content as bound oxyhemoglobin. Because the relative amount of deoxygenated blood (that is, deoxyhemoglobin) is lowered, a slight increase in signal intensity occurs because of the corresponding relative decrease in the spin-dephasing paramagnetic effects of deoxyhemoglobin. ${ }^{6}$ Although this coupling of blood oxygen changes and neuronal activation is tight and well localized, the response time is relatively slow, measured in seconds, relative to the time of neuronal activation, which is measured in tens or hundreds of milliseconds. Thus, functional tasks are designed to be repetitive (for example, finger-thumb tapping) and to take place typically over the course of 20 to 40 seconds, then cycled with "rest" periods of equal length for several cycles to increase the signal-to-noise ratio and the statistically accuracy of observed "activations." 9

Magnetic resonance imaging sequences used to create BOLD fMR imaging have evolved in line with the progression of MR imaging technology. Earlier studies were performed using standard gradient-echo 1.5-tesla acquisitions, acquired in single-section mode that involved conventional $10-\mathrm{mT} / \mathrm{m}$ gradients. The application of echo-planar imaging gradients has resulted in significant decrease in image acquisition times. Multisection-mode acquisitions with echo-planar spin echo or single-shot gradient-recalled echo-planar imaging in which high-performance gradients are used now allow even faster acquisitions. High-field 3-tesla imaging further enhances spatial resolution and signal-to-noise effects. ${ }^{7,41,42}$

\section{Task Design}

Although the task is cycled between periods of activity and rest, there is no standardization of length or number of cycles. Task cycles can vary considerably, typically from $12^{26}$ to 30 seconds, ${ }^{43}$ but must be long enough to allow the occurrence of hemodynamic changes at the cellular and macrocellular levels, while imaging data are acquired from 2.5 to 5 minutes. The data are then analyzed pixel by pixel, corrected for linear baseline drift of the MR imaging signal, and maps of cortical activity are created using statistical methods in which a correlation coefficient threshold is selected to produce a probability of a falsepositive activation due to random noise $(\mathrm{p}<0.0001$ to $\mathrm{p}<0.05$ typically) (Fig. 1). 5,17,33

Many task variants have been developed, typically to include localization of the sensory and motor cortices, Broca and Wernicke speech areas, and primary and secondary visual areas. Motor tasks have been accomplished by single finger-thumb tapping and in other cases by multiple finger-thumb tapping, self-paced clenching and spreading of the hand, or sponge squeezing. Tactile stimulation has included palm brushing, administration of air puffs to the hand, and scratching the surface of the hand. Language tasks have included picture naming by internal (silent) or vocalized speech, generating verbs, generating words in alphabetical order, listening to recordings of spoken words, or designating a category in response to an auditory presentation of nouns. ${ }^{3,15-17,24}$ Visual tasks have included intermittent photic stimulation with MR imag-

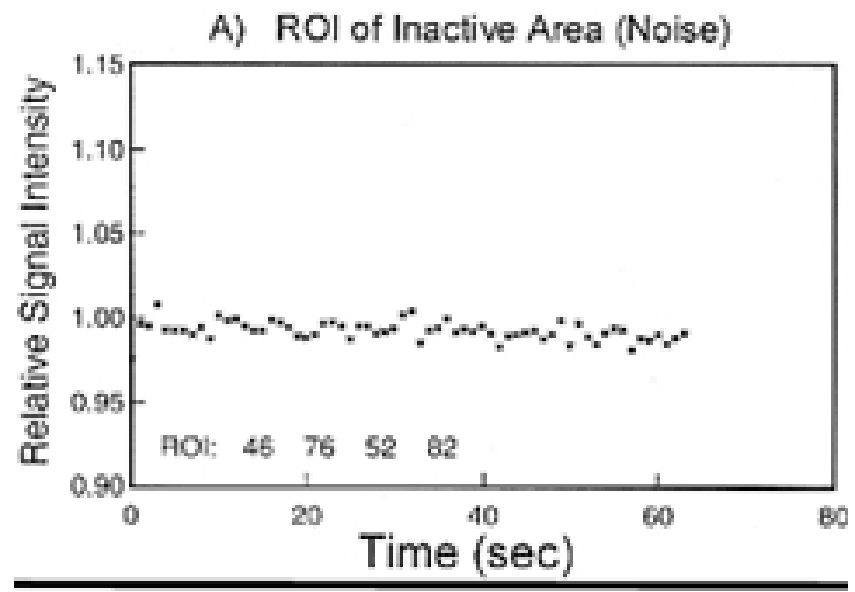

B) ROI of Active area

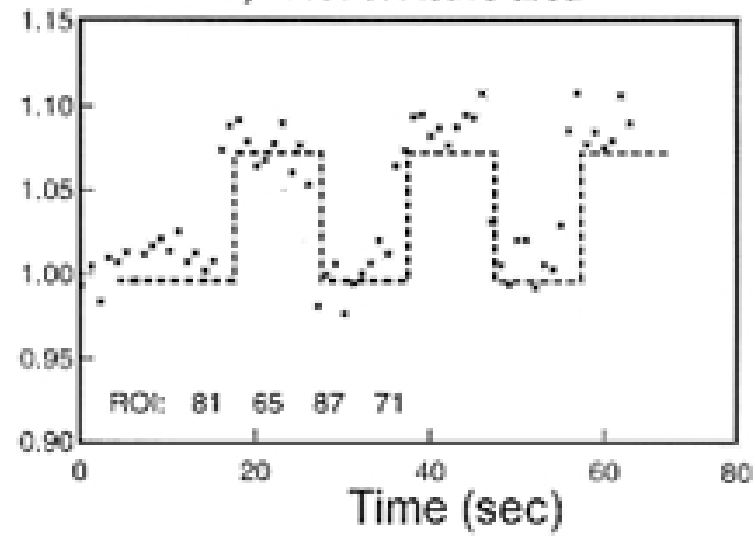

Fig. 1. Measured signal from regions of interest on unactivated area (A) and activated motor cortex (B), showing correlation between signal changes and "box-car" on-off task paradigm. $\mathrm{ROI}=$ region of interest.

ing-compatible binoculars and various projected pattern stimuli. ${ }^{26}$ Viable future directions would conceivably include task designs for memory functions, high-level cognitive tasks, and perhaps even emotion and affect. ${ }^{17}$

\section{CURRENT CONSIDERATIONS}

\section{Technical Limitations}

Venous Effects. A limitation of positive BOLD fMR imaging is that the signal is not confined to the microvasculature but spreads into veins that drain blood from the activated brain tissue, and these draining veins may be several millimeters or up to a centimeter away from the actual site of neuronal activation. Thus, the degree to which blood flow changes correlate spatially with neuronal activity is not known. Because local hemodynamic effects are the basis for BOLD fMR imaging, false-positive activity within adjacent draining veins can complicate interpretation.

Patient Motion and Compliance. The primary reason for the failure of fMR imaging examinations is patientgenerated motion. ${ }^{26}$ As examination times have decreased with faster imaging modalities, such as single-shot echo- 
planar imaging, the contribution of head motion to failed examination has decreased. Securing the head with Velcro straps, foam rubber pads or pillows, or combinations thereof have been used. ${ }^{11}$ Visual fixation crosshair has also been used to minimize head motion. ${ }^{17}$ Significant motion degradation of study quality, however, can occur simply as a result of performing the designed task. Even movements of the body away from the head can cause magnetic field distortions that can affect test accuracy. Foot or toe movement paradigms are more difficult to perform for this reason, because there is greater inadvertent transfer of movement to the rest of the body with foot or toe wiggling. Other patient compliance issues may also arise, often related to fixed neurological or cognitive deficits that may be associated with the underlying disease. Occasionally, patients are simply unable to complete a diagnostic test battery.

Limitations of Task Design. Nonstandardization of task design can potentially present a problem regarding reproducibility. For instance, as noted previously, language task designs are quite varied, and the relative sensitivity and anatomical specificity of each task design is not known. In addition, for task designs such as silent naming monitoring, the proper performance of the task may not be possible, but if vocalization is used, increased motion becomes more problematic.

\section{Interpretation of Results}

Tasks can be categorized as simple and complex. Simple tasks include simple motor tasks such as fingerthumb tapping, which typically have relatively well-defined focal areas of cortical activation, although secondary or supplemental areas exist for even some of the most apparently simple tasks, the interaction among which is poorly understood. Thus, because preoperative fMR imaging maps often demonstrate more distribution of activity than intraoperative maps, the question of which active regions are essential to the function is raised, and the possibility of false-positive activity must be considered. Complex tasks, which are even more poorly understood, include complex language and cognitive functions such as language, memory, and emotion. These tasks require both simultaneous/sequential activation and inhibition of multiple regions of the brain. ${ }^{1,3,11,36}$ Consideration of the multiple sensory inputs typical to these areas (such as both visual and auditory inputs into areas of language functionality) is an additional source of complexity.

A complicating factor in patients with brain lesions is the apparent ability of the brain to reorganize topographic functionality as a response to a pathological entity affecting an involved functional center. ${ }^{45}$ Qualitative differences were observed in the degree of bilateral activation following movement of the hand ipsilateral compared with contralateral to the side of a lesion, in which there was greater bilaterality of activation during movement of the hand corresponding to the symptomatic hemisphere (Fig. 2). ${ }^{43}$ The effects of tumor angiogenesis and vascular steal is another issue requiring further study, as are the effects of other incidental pathological entities.

\section{Sensitivity of the Tool}

Hirsch, et al., ${ }^{17}$ postulated that overall sensitivity would be improved by targeting common critical areas with multiple tasks, including functions repeated in both "active" (volitional) and "passive" (receptive) modes. This method was predicated on the existence of redundancy in functional areas of cortical activation for related tasks. It is known, for example, that functional overlap exists between the pre- and postcentral gyri for both motor function (active tapping) and sensory stimuli (passive touching). For language-related function, when the only task used was silent picture naming, the Wernicke area on the superior temporal gyrus was identified in only $73 \%$. When the task involved listening to spoken words, however, activation of this area was $100 \%$. Visual tasks designed to exploit this overlapping effect included viewing of a reversing checkerboard stimulus (passive) and viewing of pictures during a naming task that requires a response (active). Overall, administering the battery of multiple tasks in healthy volunteers, Hirsch reported $100 \%$ sensitivity for identifying the language-related cortex in the superior temporal gyrus, $100 \%$ for identifying the central sulcus and visual cortex, and 93\% in identifying the Broca area. ${ }^{17}$ Compared with the results of Wada testing for identification of hemispheric language dominance, there was $100 \%$ correlation. ${ }^{1,17}$

What of patients with brain tumors, who, along with those suffering from epilepsy, would be the target population for these procedures? Normal brain anatomy is frequently distorted by tumor mass effect and edema (Fig. 2). In 125 surgically treated patients with disease in the region of the central sulcus, Hirsch, et al. ${ }^{17}$ reported that the active area of the postcentral gyrus was identified in $94 \%$ when a tactile stimulation task was undertaken and in the precentral gyrus identified in $89 \%$ when the finger-thumb tapping paradigm was performed; overall localization of the central sulcus was possible in $97 \%$ of patients. For language functions in surgically treated patients with tumors in the specific regions, the Wernicke area was identified in $31(91 \%)$ of 34 patients and the Broca area in $17(77 \%)$ of 22 patients. Visual cortex (calcarine sulcus and inferior occipital gyrus) was identified in six of six patients with lesions in these areas. In their series, the most common cause of failure was neurological deficits such as cognitive loss or tumor-related expressive or receptive aphasias. The second-most common cause was excessive (not correctable) head motion. Marginal compliance was also cited, and false-negative results were extremely low.

\section{Localization Accuracy and Direct Electrophysiological Testing}

The spatial accuracy of fMR imaging is within $5 \mathrm{~mm}$, which is well within the approximately $10-\mathrm{mm}$ spread of electrical current to adjacent cortex known to occur when using invasive mapping modalities. Numerous investigators have corroborated the spatial specificity of fMR imaging vis-à-vis electrocortical mapping and SSEP testing for identifying simple task centers of motor and sensory function, typically within $1 \mathrm{~cm} .1,10,11,17,19,27,32,34,43,46$ Functional MR imaging also has the advantage of being able to identify activation areas deep within the sulcus that are not detectable by cortical stimulation at the brain surface. ${ }^{1}$ Agreement between fMR imaging and electrophysiological testing data, however, appears less clearcut when 

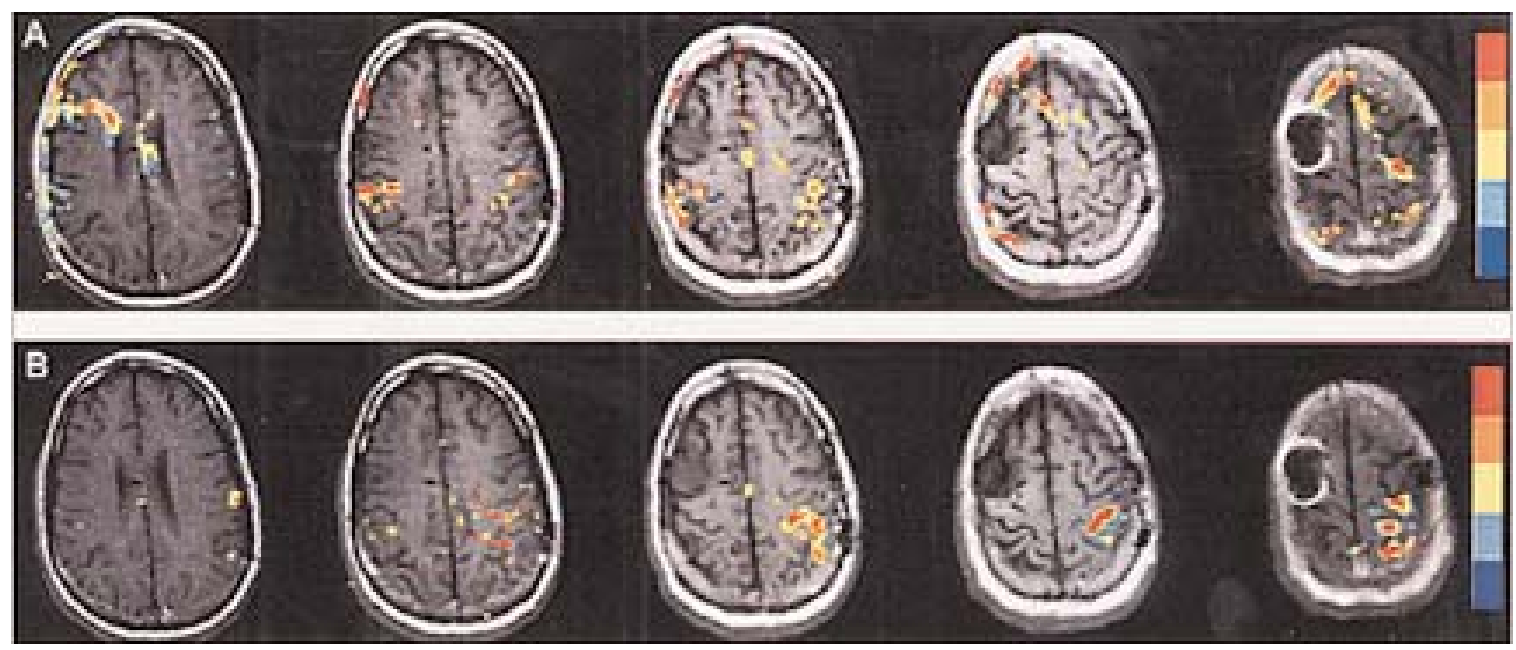

Fig. 2. Functional MR imaging studies obtained using the hand movement paradigm in a patient with a right frontal mass. There are five contiguous 8-mm transaxial slices (for each hand) covering the central sulcus. A: Colored pixels demonstrate regions activated by movements of the left hand and fingers. B: Colored pixels depict corresponding movements of the right hand and fingers. Movements of the right hand predominantly activate regions in the left hemisphere, whereas areas in both hemispheres (but more in right than left) are activated by the left hand. Note displacement of motor areas within the right hemisphere by mass effect from the tumor in first image in A

it involves complex tasks such as language. The majority of investigators have shown good correlation, particularly if a battery of language tests with functional redundancy (for example, both passive and active tasks that overlap functional areas) was built into the task design. ${ }^{11,17,36}$ Schlosser, et al. ${ }^{36}$ noted excellent spatial localization of language area when using fMR imaging, but spatial extent of language localization was less satisfying. They noted, however, that a single auditory task was used in their fMR imaging study, whereas several tasks were used intraoperatively. Additionally, because the spatial extent of fMR imaging activation is partly related to the statistical threshold at which activations are plotted, lower threshold values would yield a greater number of false-positive values and enlarge the activation area. On the other hand, Roux, et al. ${ }^{33}$ reported relatively poor correlation between fMR imaging and direct cortical stimulation data when the complex task of language function was tested, as well as relatively poor reproducibility of results when studies were repeated postoperatively. Their results may also have been affected by the use of limited task redundancy (for example, the study design did not include passive listening in addition to verb generation and naming). Sobottka, et al., ${ }^{38}$ reported similar results when comparing ${ }^{18}$ FDGPET scanning and intraoperative language testing data. In both cases, it should be noted that electrical current levels necessary to achieve language disruption in cortical stimulation testing are typically not uniform and could stimulate more distant cortex through current spread, particularly if language function lies deeper in a sulcus. What is also not clear is whether the paradigm of direct cortical testing best identifies the locus needing protection for preservation of function. Functional MR imaging delineates more areas of activation related to language and other complex functions than cortical stimulation testing, but the presumably daunting task remains of elucidating the ultimate impact of surgical disruption compared with sparing of each of these areas.

Other limitations of intraoperative testing include only unilateral testing capability, the need for a relatively larger operative exposure, high cost, lengthy intraoperative time, and significant stress on the patient. Alternatively, a two-stage procedure may be used in which a subdural grid is implanted in the first stage to allow functional electrophysiological testing to be performed postoperatively; in the second stage, resection surgery is conducted using a mapping grid generated between stages. This latter approach addresses the sometimes strong psychological burden of direct intraoperative testing for the awake patient in whom a local anesthetic agent has been used. Preoperative fMR imaging assessment can significantly reduce operative time by assisting in the design of craniotomy and surgical approach while minimizing possible brain shifts. In one series, operative time was reportedly reduced by $50 \% .^{35}$

The correlation between the repetitive task paradigms used in fMR imaging and the "single-episode" task interruption process used in direct cortical stimulation mapping is not known. It cannot necessarily be assumed, for instance, that repetitive finger-thumb tapping is equivalent to electrode stimulation of the motor homunculus, although presumably some correlation exists. What is also not conclusive is whether the intraoperative cortical stimulation paradigm for any given function necessarily reflects a better delineation of function localization than fMR imaging or other physiological mapping methods. The area of task interruption that occurs with cortical stimulation is a very small zone of less than $10 \mathrm{~mm}$ and only includes areas on the surface of the brain, whereas fMR imaging reveals activities in deeper areas. It could be argued that fMR imaging, because it provides a more anatomically global view of cerebral function, would more 
accurately depict both primary and secondary (and lowerorder) sites of function. Particularly for more complex tasks that may activate multiple and bilateral areas, fMR imaging may in some cases be a more appropriate guide for conservative resection than cortical stimulation. ${ }^{14}$

Work to date involving higher-field magnets (3-tesla) has conclusively shown a significant increase in spatial resolution and signal-to-noise ratio. ${ }^{7,41,42}$ This allows the use of higher statistical thresholds for counting true functional activations without increasing false-positive (noise) activations, which could in part address some of the aforementioned discrepancies with cortical stimulation.

\section{Clinical Application and Utility}

Although a role for fMR imaging as a substitute for invasive brain mapping remains uncertain, its clinical utility transcends its current investigational status. Surgical planning can be significantly enhanced by the incorporation of fMR imaging. ${ }^{18}$ Invasive mapping is typically performed if a proposed resection is believed preoperatively to place an eloquent area such as the motor cortex at risk. Because fMR imaging can demonstrate the proximity of eloquent areas to the proposed resection site, it can be used to identify cases in which intraoperative functional mapping will be needed. In some cases, the considerable stress, expense, and operative time added to a resection procedure can be foregone because of the preoperatively acquired fMR imaging data. In fact, Lee, et al. ${ }^{26}$ concluded in a review of their experience during a 4.5-year period that the overwhelming emphasis placed on the potential for fMR imaging to replace completely invasive mapping may be misguided because its clinical value was evident without replacing invasive mapping techniques. Overall, fMR imaging studies significantly contributed to determining the feasibility of planned resection, significantly affected surgical planning preoperatively, or selected patients for invasive functional mapping (and aided in guiding intraoperative mapping) in $89 \%$ of surgically treated patients with tumors and $91 \%$ of those with epilepsy. Of course, beyond these current beneficial effects, work to increase the clinical utility of fMR imaging independent of other methodologies is ongoing.

\section{Coregistration with Neuronavigation Systems}

Data from any of the functional imaging modalities (fMR imaging, PET, or magnetoencephalography) can be transferred to neuronavigation systems to provide direct intraoperative guidance; this is accomplished by coregistering the data with the anatomical image, either a 3D computerized tomography or MR image ${ }^{21}$ (Fig. 3). Wilkinson, et al., ${ }^{43}$ concluded that the additional information provided by fMR imaging, particularly when incorporated into a neuronavigation-guided craniotomy, was highly valuable and enabled safe resection of tumor in locations previously deemed to be too high risk when using a conventional technique without intraoperative cortical mapping. None of their patients suffered permanent neurological deficit after radical tumor debulking.

For certain tumors such as low-grade gliomas with no appreciably defined margins, the radicality of resection margins also needs to be objectively determined intraoperatively. Magnetic resonance imaging is generally unable

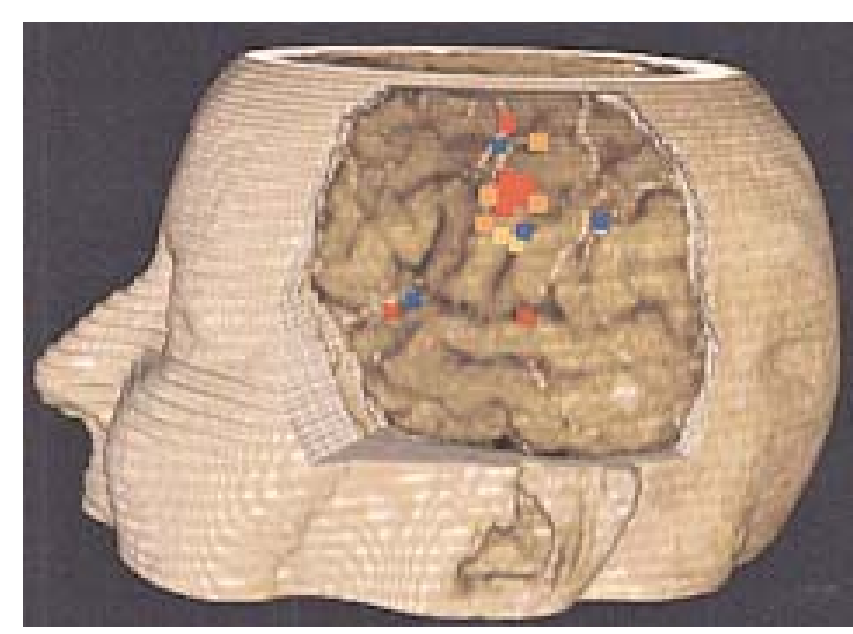

Fig. 3. Functional MR imaging data coregistered with 3D $\mathrm{T}_{1}$-weighted volumetric data set for use in intraoperative neuronavigation.

to distinguish tumor from edema. In these cases, multimodality imaging can be performed by incorporating coregistered tumor images into that of the neuronavigation system along with functional mapping data. ${ }^{35,39}$ Various agents have been used for tumor mapping, including ${ }^{18} \mathrm{FDG}-\mathrm{PET},{ }^{4,13}$ or ${ }^{11} \mathrm{C}$ methionine, ${ }^{8,29}$ or $20.1 \mathrm{Tl}$ singlephoton emission computerized tomography. ${ }^{22}$ Sabbah, et al., ${ }^{35}$ used a multimodal approach by coregistered integration of Tl SPECT tumor imaging with MR and fMR imaging mapping to optimize tumor resection.

\section{CONCLUSIONS}

Identification of eloquent areas of the brain to avoid resection-induced damage is of utmost importance for postoperative quality of life. Although the current standard for identifying these areas continues to be invasive electrophysiological testing, the development of viable noninvasive alternatives would be welcome. Invasive methods necessitate the performance of surgery in the awake patient, which can be a great strain for the patient and the surgeons in a procedure that takes several hours, and even carries the added risk of producing epileptic seizure.

Functional MR imaging has shown significant utility as an adjunctive mapping procedure and, in some cases, as a more appropriate modality for surgical guidance. If it is to become an accepted effective alternative to invasive mapping methods, however, further work must be conducted. Issues that warrant further study include the effect of tumor angiogenesis, as mentioned previously; the effects of incidental pathological findings such as atherosclerotic disease; and inter- and intraindividual variations in cerebrovascular anatomy, autoregulation, and cardiac output. Complex tasks such as language and memory present a great challenge for defining suitable testing paradigms. Nonstandard protocols and task designs as well as numerous variations in functional imaging technique represent additional confounding factors requiring study. A multimodal approach is perhaps most likely to become an effective alternative to invasive mapping. Spatial localiza- 


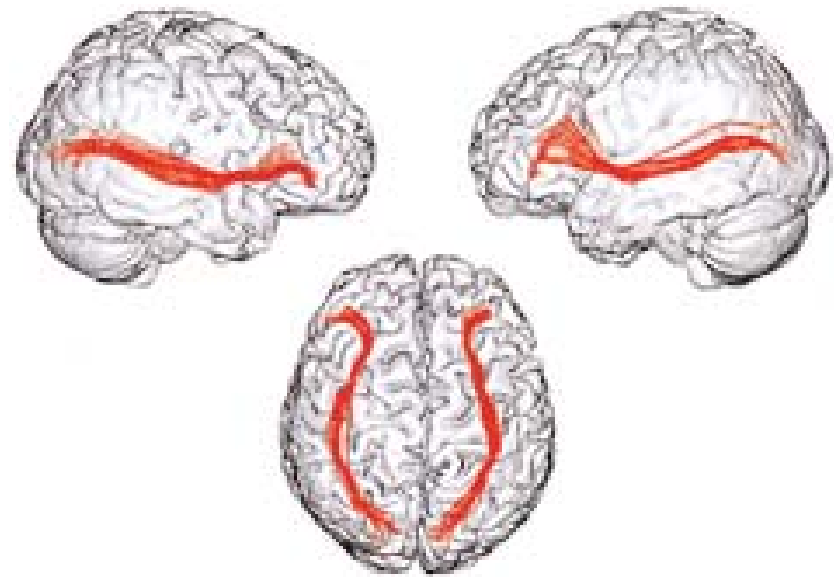

Fig. 4. Diffusion-tensor white matter tractology. A 3D diffusion-tensor image of orbitofrontal fasciculi is coregistered and fused with volume-rendered standard $\mathrm{T}_{1}$-weighted brain image.

tion issues will likely follow the routine use of high-field (3-tesla) fMR imaging.

The aforementioned standard approaches to functional mapping do not provide information about subcortical white matter. Diffusion-tensor and diffusion-weighted imaging map the directionality of water molecules at the cellular level, thus indicating the orientation of fiber tracts (Fig. 4). ${ }^{20,31,40,44}$ In one recent investigation, preoperative assessment of white matter tracts was found to be beneficial in surgical planning, allowing identification of tracts that were shifted away from the expected location and allowing for adaptation of the surgical approach to avoid destruction of these tracts. ${ }^{44}$

\section{References}

1. Bahn MM, Lin W, Silbergeld DL, et al: Localization of language cortices by functional MR imaging compared with intracarotid amobarbital hemispheric sedation. AJR 169: 575-579, 1997

2. Bandettini PA, Wong EC, Hinks RS, et al: Time course EPI of human brain function during task activation. Magn Reson Med 25:390-397, 1992

3. Binder JR, Swanson SJ, Hammeke TA, et al: Determination of language dominance using functional MRI: a comparison with the Wada test. Neurology 46:978-984, 1996

4. Bittar RG, Olivier A, Sadikot AF, et al: Presurgical motor and somatosensory cortex mapping with functional magnetic resonance imaging and positron emission tomography. J Neurosurg 91:915-921, 1999

5. Bullmore E, Brammer M, Williams SC, et al: Statistical methods of estimation and inference for functional MR image analysis. Magn Reson Med 35:261-277, 1996

6. Chen W, Ogawa S: Principles of BOLD-functional MRI, in Moonen CTW, Bandettini PA, (eds): Functional MRI. Berlin: Springer-Verlag, 2000 pp 103-114

7. Chen W, Ugurbil K: High spatial resolution functional magnetic resonance imaging at very-high-magnetic field. Top Magn Reson Imaging 10:63-78, 1999

8. De Reuck J, Santens P, Goethals P, et al: [Methyl-11C]thymidine positron emission tomography in tumoral and non-tumoral cerebral lesions. Acta Neurol Belg 99:118-125, 1999

9. Elster AD, Burdette JH: Questions \& Answers in Magnetic Resonance Imaging, ed 2. St Louis: Mosby, 2001, pp 273-280
10. Fandino J, Kollias SS, Weiser HG, et al: Intraoperative validation of functional magnetic resonance imaging and cortical reorganization patterns in patients with brain tumors involving the primary motor cortex. J Neurosurg 91:238-250, 1999

11. FitzGerald DB, Cosgrove GR, Ronner S, et al: Location of language in the cortex: a comparison between functional MR imaging and electrocortical stimulation. AJNR 18:1529-1539, 1997

12. George JS, Aine CJ, Mosher JC, et al: Mapping function in the human brain with magnetoencephalography, anatomical magnetic resonance imaging, and functional magnetic resonance imaging. J Clin Neurophysiol 12:406-431, 1995

13. Gupta NC, Nicholson P, Bloomfield SM: FDG-PET in the staging work-up of patients with suspected intracranial metastatic tumors. Ann Surg 230:202-206, 1999

14. Heilbrun MP, Lee JN, Alvord L: Practical application of fMRI for surgical planning. Stereotact Funct Neurosurg 76: 168-174, 2001

15. Herholz K, Reulen HJ, von Stockhausen HM, et al: Preoperative activation and intraoperative stimulation of languagerelated areas in patients with glioma. Neurosurgery 41: 1253-1262, 1997

16. Hinke RM, Hu X, Stillman AE, et al: Functional magnetic resonance imaging of Broca's area during internal speech. Neuroreport 4:675-678, 1993

17. Hirsch J, Ruge MI, Kim KH, et al: An integrated functional magnetic resonance imaging procedure for preoperative mapping of cortical areas associated with Tactile, motor, language, and visual functions. Neurosurgery 47:711-722, 2000

18. Jack CR, Lee CC, Ward HA, et al: The role of functional MRI in planning perirolandic surgery, in Moonen CTW, Bandettini PA, (eds): Functional MRI. Berlin: Springer-Verlag, 2000, pp 539-550

19. Jack CR Jr, Thompson RM, Butts RK, et al: Sensory motor cortex: correlation of presurgical mapping with functional MR imaging and invasive cortical mapping. Radiology 190:85-92, 1994

20. Jones DK, Simmons A, Williams SC, et al: Non-invasive assessment of axonal fiber connectivity in the human brain via diffusion tensor MRI. Magn Reson Med 42:37-41, 1999

21. Kamada K, Houkin K, Takeuchi F, et al: Visualization of the eloquent motor system by integration of MEG, functional, and anisotropic diffusion-weighted MRI in functional neuronavigation. Surg Neurol 59:352-362, 2003

22. Kim KT, Black KL, Marciano D, et al: Thallium-201 SPECT imaging of brain tumors: methods and results. J Nucl Med 31: 965-969, 1990

23. Kwong KK, Belliveau JW, Chesler DA, et al: Dynamic magnetic resonance imaging of human brain activity during primary sensory stimulation. Proc Natl Acad Sci USA 89:5675-5679, 1992

24. Latchaw RE, HU X, Ugurbil K, et al: Functional magnetic resonance imaging as a management tool for cerebral arteriovenous malformations. Neurosurgery 37:619-626, 1998

25. Lee CC, Jack CR Jr, Riederer SJ: Mapping of the central sulcus with functional MR: active versus passive activation tasks. AJNR 19:847-852, 1998

26. Lee CC, Ward HA, Sharbrough FW, et al: Assessment of functional MR imaging in neurosurgical planning. AJNR 20: 1511-1519, 1999

27. Lehericy S, Duffau H, Cornu P, et al: Correspondence between functional magnetic resonance imaging somatotopy and individual brain anatomy of the central region: comparison with intraoperative stimulation in patients with brain tumors. J Neurosurg 92:589-598, 2000

28. Mueller WM, Yetkin FZ, Hammeke TA, et al: Functional magnetic resonance imaging mapping of the motor cortex in patients with cerebral tumors. Neurosurgery 39:515-521, 1996

29. Nariai T, Senda M, Ishii K, et al: Three-dimensional imaging of 
cortical structure, function and glioma for tumor resection. J Nucl Med 38:1563-1568, 1997

30. Ogawa S, Tank DW, Menon R, et al: Intrinsic signal changes accompanying sensory stimulation: functional brain mapping with magnetic resonance imaging. Proc Natl Acad Sci USA 89:5951-5955, 1992

31. Pajevic S, Pierpaoli C: Color schemes to represent the orientation of anisotropic tissues from diffusion tensor data: application to white matter fiber tract mapping in the human brain. Magn Reson Med 42:526-540, 1999

32. Puce A, Constable T, Luby ML, et al: Functional magnetic resonance imaging of sensory and motor cortex: comparison with electrophysiological localization. J Neurosurg 83:262-270, 1995

33. Roux FE, Boulanouar K, Lotterie JA, et al: Language functional magnetic resonance imaging in preoperative assessment of language areas: correlation with direct cortical stimulation. Neurosurgery 52:1335-1347, 2003

34. Roux FE, Boulanouar K, Ranjeva JP, et al: Cortical intraoperative stimulation in brain tumors as a tool to evaluate spatial data from motor functional MRI. Invest Radiol 34:225-229, 1999

35. Sabbah P, Foehrenbach H, Dutertre G, et al: Multimodal anatomic, functional, and metabolic brain imaging for tumor resection. Clin Imaging 26:6-12, 2002

36. Schlosser MJ, Luby M, Spencer DD, et al: Comparative localization of auditory comprehension by using functional magnetic resonance imaging and cortical stimulation. J Neurosurg 91: 626-635, 1999

37. Schulder M, Maldjian JA, Liu WC, et al: Functional imageguided surgery of intracranial tumors located in or near the sensorimotor cortex. J Neurosurg 89:412-418, 1998

38. Sobottka SB, Bredow J, Beuthien-Baumann B, et al: Comparison of functional brain PET images and intraoperative brain-mapping data using image-guided surgery. Comput Aided Surg 7:317-325, 2002

39. Steinmeier R, Sobottka SB, Reiss G, et al: Surgery of low-grade gliomas near speech-eloquent regions: brainmapping versus preoperative functional imaging. Onkologie 25:552-557, 2002

40. Tamura H, Takahashi S, Kurihara N, et al: Practical visualization of internal structure of white matter for image interpretation: staining a spin-echo T2-weighted image with three echo-planar diffusion-weighted images. Am J Neurorad 24: 401-409, 2003

41. Thulborn KR: Clinical rationale for very-high-field (3.0 Tesla) functional magnetic resonance imaging. Top Magn Reson Imaging 10:37-50, 1999

42. Thulborn KR, Chang SY, Shen GX, et al: High-resolution echoplanar fMRI of human visual cortex at 3.0 tesla. NMR Biomed 10:183-190, 1997

43. Wilkinson ID, Romanowski CAJ, Jellinek DA, et al: Motor functional MRI for pre-operative and intraoperative neurosurgical guidance. Br J Radiol 76:98-103, 2003

44. Witwer BP, Moftakhar R, Hasan KM, et al: Diffusion-tensor imaging of white matter tracts in patients with cerebral neoplasm. J Neurosurg 97:568-575, 2002

45. Wunderlich G, Knorr U, Herzog H, et al: Precentral glioma location determines the displacement of cortical hand representation. Neurosurgery 42:18-27, 1998

46. Yetkin FZ, Mueller WM, Morris GL, et al: Functional MR activation correlated with intraoperative cortical mapping. AJNR 18:1311-1315, 1997

47. Yousry TA, Schmid UD, Jassoy AG, et al: Topography of the cortical motor hand area: prospective study with functional MR imaging and direct motor mapping at surgery. Radiology 195: 23-29, 1995

Manuscript received June 11, 2003

Accepted in final form June 20, 2003.

Address reprint requests to: Paul E. Kim, M.D., University of Southern California Keck School of Medicine, 1200 North State Street, Room 3740, Los Angeles, CA 90033. email: pek@usc.edu. 